\title{
PARÁMETROS DE EVALUACIÓN DIFERENCIADOS PARA LA ECOGRAFÍA OBSTÉTRICA ESTANDAR VERSUS LA ESPECIALIZADA RECOMENDACIÓN DEL SERVICIO DE MEDICINA FETAL 2019
}

\author{
DIFFERENTIATED EVALUATION PARAMETERS FOR THE STANDARD OBSTETRIC \\ ECOGRAPHY VERSUS THE SPECIALIZED \\ RECOMMENDATION OF THE FETAL MEDICINE SERVICE 2019
}

\author{
Walter Castillo Urquiaga ${ }^{1 a}$, Walter R. Ventura Laveriano ${ }^{1 a}$, Oscar A. Limay Rios ${ }^{1 a}$, Mario I. Zárate Girao ${ }^{1 a}$, \\ Raul R. Sugajara Rosario ${ }^{1 a}$, Cecilia E. Ibáñez Rodríguez ${ }^{1 a}$, Oswaldo M. Gonzáles Carrillo ${ }^{1 a}$.
}

\section{INTRODUCCIÓN}

El presente documento resulta del análisis del ejercicio de la ultrasonografía obstétrica en el Perú y como un aporte para el ordenamiento que repercuta favorablemente en la calidad de las evaluaciones y en la salud materno perinatal considerando, adoptando y adaptando normativas y lineamientos internacionales de entidades referentes en ecografía obstétrica y medicina fetal.

Según consta en la Guía clínica de Ultrasonografía en el embarazo del 2016, diversas sociedades referentes han adoptado la siguiente terminología uniforme para tres tipos de exámenes ecográficos según nivel de complejidad: Estandar, Limitada y Especializada. Algunas de tipo "especializada", como las ecografías de tamizaje "Genética y Morfológica" no significa que deba ser realizada necesariamente por un médico especialista o subespecialista; sino que quien la realice deba tener la competencia requerida para una evaluación de tal complejidad.

Además, al revisar los Protocolos de organizaciones internacionales como The Fetal Medicine Foundation de Londres (FMF), The Society for Maternal Fetal Medicine (SMFM), International Society of Ultrasound in Obstetrics and Gynecology (ISUOG), la Guía nacional Técnica de Ecografía Básica Obstétrica y Ginecológica INMP 2009 y la Guía de práctica clínica y de procedimientos en obstetricia y ginecología INMP 2018 advertimos inconsistencias y diferencias en las descripciones y denominaciones de los diferentes tipos de ecografía que deben actualizarse, estandarizarse e implementarse. La denominación de ecografía "genética" a la ecografía de "tamizaje, screening o nuchal scan" del primer trimestre (término en países desarrollados) debe su nombre al traslado del término "genético" desde el segundo hacia el primer trimestre dada la mayor posibilidad de visualizar al feto y por ende evidenciar anomalías o marcadores genéticos y/o cromosómicos. Es una denominación que ya está arraigada en nuestro país y en algunas partes del mundo ("first trimester genetic ultrasound) pero sin parámetros de evaluación uniformes ni diferenciación en niveles de complejidad.

Parte de la problemática de la salud pública materno perinatal se explica por el no acceso de un porcentaje de gestantes a evaluaciones ecográficas de calidad debido principalmente a la ausencia de parámetros de evaluación generando gastos innecesarios, impacto emocional, y desenlaces adversos o complicaciones no detectadas ni evitadas. Muchas evaluaciones ecográficas no cumplen los parámetros para su denominación como especializada y muchas otras no cumplen ni los parámetros básicos. Esto amerita la unificación y difusión de parámetros como tipo de ecografía según complejidad, edad a realizarse, información a obtener, competencias del evaluador, objetivos de evaluación, modo ecográfico, vía de abordaje y duración.

En el Perú, el ejercicio de la ultrasonografía es desordenado sin un ente normativo y rector que conduzca formalmente un proceso de certificación, acreditación, auditoría y evaluación de los médicos ecografistas. Según normativas internacionales, el sonologist (physician o médico especialista en ecografía materno fetal) es el único facultado no solo para realizar el procedimiento sino para interpretar y reportar el informe final o diagnóstico y debe

Instituto Nacional Materno Perinatal. Lima, Perú.

Médico Gíneco-Obstetra. Servicio de Medicina Fetal. Lima-Perú.

Citar como: Castillo W, Ventura WR, Limay OA, Zárate MI, Sugajara RR, Ibañez CE, Gonzáles OM. Parámetros de evaluación diferenciados para la ecografía obstétrica estandar versus la especializada. Recomendación del Servicio de Medicina Fetal 2019. Rev Peru Investig Matern Perinat $2019 ;$ 8(1):49-55. DOI: https://doi.org/10.33421/inmp.2019144 
supervisar y refrendar la evaluación del sonographer o no médico, en los países donde la práctica de este último está regulada.

Ante la ausencia oficial nacional de un ente rector en el ejercicio de la ecografía, el Servicio de Medicina Fetal del INMP viene considerando desde hace años estos estándares internacionales en las diferentes evaluaciones que realiza, pero en una forma no protocolizada objetivamente.

En este contexto, recomendamos estos parámetros de evaluación para referencia institucional y extra institucional fomentando la certificación y acreditación con calidad semejante a la Fetal Medicine Foundation.

\section{TIPOS Y CRITERIOS DE ECOGRAFÍA OBSTÉTRICA ESTANDAR (SIMPLE- BÁSICA - BAJA COMPLEJIDAD - ATENCIÓN PRIMARIA - PRIMER NIVEL)}

\section{Componentes mínimos de una evaluación ecográfica estándar:}

a. Determinación inicial de gestación.

b. Determinación de presentación y número de sacos gestacionales, placenta o fetos.

c. Determinación del volumen de líquido amniótico.

d. Determinación de la actividad cardíaca.

e. Determinación de la ubicación placentaria.

f. Medición biométrica (CC, CA, DBP y LF) con estimación del peso fetal y su percentil (según tabla de Hadlock) en relación a la edad gestacional real para evaluar el crecimiento.

g. Evaluación anatómica básica o rutinaria de la anatomía fetal y del contenido uterino durante el 1er, 2do y 3er trimestre (Ver Tabla). Esta evaluación es diferente y de menor complejidad que la evaluación ecográfica obstétrica especializada: Genética y Morfológica (evaluación anatómica detallada con marcadores de cromosomopatías: Genetic scan del primer o segundo trimestre).

h. Determinar necesidad de evaluación complementaria y referir para examen ecográfico de mayor complejidad en áreas especializadas como Medicina Fetal.

Quien debe realizar las evaluaciones ecográficas estándar:

\section{Prioritariamente:}

- Médico especialista en Radiología e imágenes capacitado, certificado y con experiencia para ecografía obstétrica estándar o básica. El médico con capacidad para realizar la ecografía especializada también puede realizar una evaluación básica.

\section{Secundariamente:}

- Médico especialista en ginecobstetricia capacitado, certificado y con experiencia para ecografía obstétrica estándar o básica.

- Médico general capacitado, certificado y con experiencia para ecografía obstétrica estándar o básica.

\subsection{ECOGRAFÍA OBSTÉTRICA BÁSICA DEL 1ER TRIMESTRE TRANSVAGINAL:}

- Tipo de Ecografía: Estándar (Básica, Primaria o 1 er Nivel).

- Edad a realizarse: Menor de 11 semanas, embarazo temprano.

- Informa: Actividad cardiaca o viabilidad, edad gestacional. Embarazo único o múltiple. Determinar necesidad de evaluación complementaria y referir para examen ecográfico de mayor complejidad.

- Evaluador: Médico especialista en Radiología, Ginecobstetricia o Médico General capacitado, certificado y con experiencia para ecografía obstétrica estándar o básica transvaginal.

- Evalúa:

- Latido cardiaco.

o Localización del embarazo; intrauterino versus ectópico.

- Biometría de LCN (Longitud Corono Nalga) y precisión de la edad gestacional.

o Evaluación básica embrionaria; número y apariencia.

o Saco gestacional y saco vitelino; número y apariencia.

- Normalidad del útero y anexos

o Sospecha de gestación inviable o de localización desconocida

- Modo ecográfico: 2D bidimensional. Modo M para la frecuencia cardiaca.

- Abordaje: Transvaginal.

- Duración: Aproximadamente 5 minutos

\subsection{ECOGRAFÍA OBSTÉTRICA BÁSICA DE 11 a 14} SEMANAS TRANSABDOMINAL:

- Tipo de Ecografía: Estándar (Básica, Primaria o 1er Nivel).

- Edad a realizarse: De de 11 a 14 semanas

- Informa: Actividad cardiaca, edad gestacional y número de fetos. Determina normalidad de feto, placenta y líquido y necesidad de evaluación complementaria o referencia para examen ecográfico de mayor complejidad.

- Evaluador: Médico especialista en Radiología, Ginecobstetricia o Médico General capacitado, certificado y con experiencia para ecografía obstétrica estándar o básica transabdominal.

- Evalúa:

- Latido cardiaco y número de fetos.

o Biometría de LCN (Longitud Corono Nalga) 
o DBP (Diámetro Biparietal) y precisión de la edad gestacional.

- Evaluación anatómica fetal básica no detallada de algunos órganos como cabeza, abdomen y pared abdominal.

- Normalidad de líquido y placenta.

- Modo ecográfico: 2D bidimensional. Modo M o Doppler para la frecuencia cardiaca.

- Abordaje: Transabdominal.

- Duración: Aproximadamente 15 minutos si es un solo feto

\subsection{ECOGRAFÍA OBSTÉTRICA BÁSICA DEL} 2DO/3ER TRIMESTRE TRANSABDOMINAL:

- Tipo de Ecografía: Estándar (Básica, Primaria o 1 er Nivel).

- Edad a realizarse: Mayor de 14 semanas

- Informa: Actividad cardiaca, estimación del peso y su percentil de crecimiento en relación con la edad gestacional. Determina normalidad de anatomía fetal básica, de placenta y líquido y necesidad de evaluación complementaria o referencia para examen ecográfico de mayor complejidad.

- Evaluador: Médico especialista en Radiología, Ginecobstetricia o Médico General capacitado, certificado y con experiencia para ecografía obstétrica estándar o básica transabdominal.

\section{- Evalúa:}

- Latido cardiaco, posición y presentación fetal.

- Biometría de DBP-CC-CA-LF, estimación del peso fetal y su percentil en relación a la edad gestacional

- En ausencia de ecografía precoz, ayuda a precisar la edad gestacional.

- Evaluación anatómica fetal básica no detallada.

o Normalidad, localización y grado placentario.

- Normalidad de líquido amniótico, ILA desde las 20 semanas.

- Modo ecográfico: 2D-bidimensional. Modo M o Doppler para la frecuencia cardiaca fetal.

- Abordaje: Transabdominal.

- Duración: Aproximadamente 20 minutos si es un solo feto.

\section{ECOGRAFÍAS OBSTÉTRICAS ESPECIALIZADAS (COMPLEJA-AVANZADA- DETALLADA- 3ER NIVEL)}

Componentes mínimos de una evaluación ecográfica especializada: Lo obtenido en el examen estándar más:

a. Tamizaje o predicción de síndromes cromosómicos, Pre eclampsia, RCIU y otras complicaciones materno fetales. b. Evaluación de marcadores de cromosomopatías y diagnóstico y seguimiento de patología fetal o placentaria.

c. Evaluación anatómica detallada por sistemas orgánicos

d. Evaluación Doppler de corazón, vasos y de la función cardiaca

e. Evaluación biofísica del bienestar fetal

f. Medidas biométricas adicionales

g. Evaluación del embarazo múltiple, corionicidad y amnionicidad

h. Toda evaluación ecográfica de alto riesgo materno perinatal como en sospecha, evidencia o seguimiento de complicación fetal primaria o secundaria a morbilidad placentaria o materna: Malformaciones fetales y placentarias, alteraciones del crecimiento fetal, embarazo múltiple, trastornos hipertensivos y morbilidades maternas.

\section{Quién debe realizar las evaluaciones ecográficas} especializadas:

\section{Prioritariamente:}

- Médico especialista en ginecobstetricia con subespecialidad, fellowship, o capacitación de pos grado en medicina materno fetal que labora exclusivamente en un area de medicina materno fetal. Éste también tiene las competencias para las evaluaciones ecográficas básicas.

\section{Secundariamente:}

- Médico especialista en ginecobstetricia, Médico radiólogo o Médico general capacitados y acreditados mediante certificado de competencia o auditados por entidades como Fetal Medicine Foundation con experiencia en ecografía obstétrica especializada de tamizaje y quien debe referir al especialista en evaluación fetal ante la duda o anormalidad.

Al ser una evaluación especializada con conocimiento y técnicas avanzadas exige que el evaluador sea capacitado, con experiencia y capacidad para interpretar, comunicar, reportar, supervisar, sugerir manejos y pronósticos o identifique la necesidad de un manejo sub especializado.

\subsection{ECOGRAFÍA GENÉTICA}

- Tipo de Ecografía: Especializada (Avanzada o 3er Nivel) de tamizaje.

- Edad a realizarse: 11 a 13 semanas + 6 días.

- Informa: Riesgo de alteraciones cromosómicas/genéticas a través de marcadores, Anomalías estructurales detectables a esta edad, corionicidad, predice Restricción del crecimiento intrauterino (RCIU) y Preeclampsia y brinda identificación inicial de gestantes de alto riesgo y bajo riesgo con mayor seguridad 
inicial de normalidad como un concepto moderno de calidad.

- Evaluador: Médico especialista en ginecobstetricia con subespecialidad, fellowship, o capacitación de pos grado en medicina materno fetal que labora exclusivamente en un area de medicina materno fetal con competencia para el cálculo del riesgo, interpretación y manejo; Ginecobstetra, Radiólogo o médico general capacitados y acreditados mediante certificado de competencia o auditados por entidades como Fetal Medicine Foundation con experiencia en ecografía obstétrica especializada quienes deben referir a medicina fetal en caso de duda o anormalidad.

- Evalúa:

- Marcadores de cromosomopatía: Translucencia nucal, Hueso nasal, Ductus venoso, Flujo tricuspideo.

- Biometría y crecimiento fetal temprano

- Evaluación anatómica fetal básica de todos los órganos con evaluación detallada de algunos órganos evaluables a esta edad.

- Precisión de la edad gestacional.

- Corionicidad en embarazo múltiple.

- Doppler de Arterias Uterinas

- Modo ecográfico:

- 2D para biometría, crecimiento fetal temprano y precisión de la edad.

- Doppler para evaluación de Ductus Venoso, Flujo Tricuspideo, Arterias Uterinas y corazón.

o 3D-5D, opcionalmente, para evaluación anatómica, anomalías uterinas o lesión placentaria.

- Abordaje: Generalmente transabdominal y complementariamente transvaginal, para la evaluación anatómica precoz y opcionalmente, cervicometría.

- Duración: Aproximadamente 40 minutos si es un solo feto.

\subsection{ECOGRAFÍA MORFOLÓGICA}

\section{Evaluación anatómica detallada del feto.}

- Tipo de Ecografía: Especializada (Avanzada o 3er Nivel) de tamizaje.

- Edad a realizarse: Generalmente entre las 20 a 24 semanas

- Informa: Riesgo de alteraciones cromosómicas/ genéticas a través de marcadores, predice Restricción del crecimiento intrauterino (RCIU) y Preeclampsia, detecta anomalías estructurales, riesgo de parto prematuro y brinda identificación inicial de gestantes de alto riesgo y bajo riesgo con mayor seguridad inicial de normalidad como un concepto moderno de calidad.
- Evaluador: Médico especialista en ginecobstetricia con subespecialidad, fellowship, o capacitación de pos grado en medicina materno fetal que labora exclusivamente en un area de medicina materno fetal con competencia para el cálculo del riesgo, interpretación y manejo; Ginecobstetra, Radiólogo o médico general capacitados y acreditados mediante certificado de competencia o auditados por entidades como Fetal Medicine Foundation con experiencia en ecografía obstétrica especializada quienes deben referir a medicina fetal en caso de duda o anormalidad.

- Evalúa:

- Marcadores de cromosomopatía: Pliegue nucal, Hueso nasal, Húmero corto, Intestino hiperecogénico, Ventriculomegalia, otros marcadores.

- Biometría y crecimiento fetal

- Evaluación anatómica detallada e identificación de anomalías detectables

o Precisión de la edad gestacional.

- Doppler de Arterias Uterinas

- Cervicometría

- Modo ecográfico:

- 2D para biometría, crecimiento fetal y precisión de la edad.

o Doppler para evaluación de Arterias Uterinas y el corazón.

o 3D-5D, opcionalmente, para evaluación anatómica, anomalías uterinas o lesión placentaria

- Abordaje: Transabdominal y complementariamente transvaginal, para la evaluación de ubicación placentaria y cervicometría.

- Duración: Aproximadamente 40 minutos si es un solo feto.

\subsection{ECOGRAFÍA DOPPLER OBSTÉTRICO}

\section{Evaluación hemodinámica del bienestar fetal.}

- Tipo de Ecografía: Especializada (Avanzada o 3er Nivel).

- Edad a realizarse: Mayor a las 22 semanas, después de la etapa de aborto.

- Informa: Optimiza la predicción, el diagnóstico, la severidad o grado de compromiso, el seguimiento y manejo del feto con Restricción del crecimiento intrauterino (RCIU) y Preeclampsia. Brinda identificación de gestantes de alto riesgo y bajo riesgo como un concepto moderno de calidad. Optimiza la evaluación cardiovascular y el diagnóstico, seguimiento y manejo de malformaciones o complicaciones materno fetales por el especialista. Signos de anemia fetal o riesgo de 
muerte perinatal por insuficiencia placentaria primaria o secundaria a condiciones maternas.

- Evaluador: Médico especialista en ginecobstetricia con subespecialidad, fellowship, o capacitación de pos grado en medicina materno fetal que labora exclusivamente en un area de medicina materno fetal con competencia para el diagnóstico, interpretación y manejo; Ginecobstetra, Radiólogo o médico general capacitados y acreditados mediante certificado de competencia o auditados por entidades como Fetal Medicine Foundation con experiencia en ecografía obstétrica especializada quienes deben referir a medicina fetal en caso de duda o anormalidad.

- Evalúa:

- Resistencia de vasos como Arterias Uterinas, Arteria Umbilical, A. Cerebral Media, Ductus venoso, Istmo Aórtico, etc.

- Velocidad de Pico Sistólico de la A. Cerebral Media

- Evaluación integrada del bienestar con el Perfil Biofísico

- Biometría y evaluación del crecimiento fetal

- Revaluación anatómica e identificación de anomalías detectables

- Signos de acretismo placentario, hematomas o lesiones placentarias

- Doppler de Arterias Uterinas

- Modo ecográfico:

- 2D para biometría, crecimiento fetal y precisión de la edad.

- Doppler para evaluación de vasos periféricos fetales, vasos maternos y el corazón fetal.

o 3D-5D, opcionalmente, para evaluación anatómica vascular e irrigación uterina y placentaria.

- Abordaje: Generalmente transabdominal y complementariamente transvaginal, para la evaluación anatómica y placentaria.

- Duración: Aproximadamente 30 minutos si es un solo feto.

\subsection{PERFIL BIOFÍSICO FETAL}

\section{Evaluación ecográfica del bienestar fetal.}

- Tipo de Ecografía: Especializada (Avanzada o 3er Nivel).

- Edad a realizarse: Generalmente a partir de las 28 semanas.

- Informa: Estado del bienestar fetal a través de indicadores biofísicos que se alteran en situaciones de hipoxia, infecciones, hemorragias, etc.

- Evaluador: Médico especialista en ginecobstetricia con subespecialidad, fellowship, o capacitación de pos grado en medicina materno fetal que labora exclusivamente en un area de medicina materno fetal con competencia para la evaluación, interpretación y manejo; Ginecobstetra, Radiólogo o médico general capacitados y acreditados mediante certificado de competencia o auditados por entidades como Fetal Medicine Foundation con experiencia en ecografía obstétrica especializada quienes deben referir a medicina fetal y/o a emergencia en caso de duda o anormalidad de alguno de los parámetros.

- Evalúa:

- Evaluación del tono fetal.

- Evaluación del mvimiento corporal fetal.

o Evaluación del movimiento respiratorio fetal.

- Evaluación del volúmen del líquido amniótico con ILA.

- Evaluación de la reactividad fetal.

- Modo ecográfico:

- 2D para biometría, crecimiento.

- 2D para evaluación del perfil biofísico

- Doppler, opcionalmente, para evaluación de pozos de líquido y no ser confundidos con cordón umbilical.

- Abordaje: Transabdominal.

- Duración: Aproximadamente 30 minutos si es un solo feto

\subsection{NEUROSONOGRAFÍA FETAL}

\section{Evaluación del sistema nervioso fetal.}

- Tipo de Ecografía: Especializada (Avanzada o 3er Nivel).

- Edad a realizarse: Generalmente a partir de las 20 semanas.

- Informa: Normalidad, anormalidad o precisión diagnóstica, pronostica y manejo de una anomalía estructural o vascular del sistema nervioso central y periférico del feto.

- Evaluador: Médico especialista en ginecobstetricia con subespecialidad, fellowship, o capacitación de pos grado en medicina materno fetal que labora exclusivamente en un area de medicina materno fetal con competencia para la evaluación, interpretación, diagnóstico y manejo.

- Evalúa:

- Evaluación anatómica detallada de cráneo y cerebro.

- Biometría y crecimiento del cráneo y del feto

- Evaluación anatómica detallada de columna

- Evaluación anatómica integrada del feto

- Modo ecográfico:

- 2D para biometría, crecimiento y anatomía.

- Doppler para evaluación de Arterias Cerebrales. 
o 3D-5D, opcionalmente, para evaluación anatómica y de anomalías en múltiples planos o evaluación vascular

- Abordaje: Transabdominal y complementariamente transvaginal, para la evaluación bidimensional o tridimensional del cerebro fetal.

- Duración: Aproximadamente 50 minutos si es un solo feto

\subsection{ECOCARDIOGRAFÍA FETAL Y FUNCIÓN CARDIACA}

\section{Evaluación del sistema cardiovascuar fetal.}

- Tipo de Ecografía: Especializada (Avanzada o 3er Nivel).

- Edad a realizarse: Generalmente a partir de las 20 semanas. Opcionalmente entre las 12 a 14 semanas por alguna indicación.

- Informa: Normalidad, anormalidad o precisión diagnóstica, pronóstica y manejo de una anomalía estructural, vascular o funcional del sistema cardiovascular del feto por condición primaria o secundaria a trastorno fetal 0 materno.

- Evaluador: Médico especialista en ginecobstetricia con subespecialidad, fellowship, o capacitación de pos grado en medicina materno fetal que labora exclusivamente en un area de medicina materno fetal con competencia para la evaluación, interpretación, diagnóstico y manejo.

- Evalúa:

- Evaluación anatómica detallada del corazón.

- Biometría de corazón, cámaras cardiacas y vasos

- Evaluación vascular de vasos periféricos

- Evaluación del ritmo cardiaco y el diagnóstico, pronóstico y manejo de sus anormalidades.

- Evaluación de la función cardiaca, repercusion y manejo.

- Diagnóstico, pronóstico y manejo de malformaciones cardiacas primarias o secundarias.

- Modo ecográfico:

- 2D para biometría, crecimiento y anatomía.

o Doppler para evaluación de cavidades, tractos de salida, grandes vasos y flujos periféricos.

o 3D-5D, opcionalmente, para evaluación anatómica y de anomalías en múltiples planos o evaluación vascular

- Abordaje: Transabdominal complementariamente transvaginal, evaluación bidimensional o tridimensional del corazón fetal, en especial en el primer trimestre.
- Duración: Aproximadamente 50 minutos si es un solo feto.

\subsection{ECOGRAFÍA 3D/4D}

Evaluación tridimensional estructural o vascular del feto y anexos fetales.

- Tipo de Ecografía: Especializada (Avanzada o 3er Nivel).

- Edad a realizarse: Con fines diagnósticos, en cualquier trimestre del embarazo.

- Informa: Normalidad, anormalidad o precisión diagnóstica, pronóstica y manejo de una anomalía estructural o vascular de la anatomía fetal.

- Evaluador: Médico especialista en ginecobstetricia con subespecialidad, fellowship, o capacitación de pos grado en medicina materno fetal que labora exclusivamente en un area de medicina materno fetal con competencia para la evaluación, interpretación, diagnóstico y manejo.

- Evalúa:

- Evaluación anatómica 3D/4D detallada de cráneo y cerebro, corazón y estructuras embrionarias.

o Evaluación anatómica detallada 3D/4D de miembros, cara y toda la superficie fetal.

o Evaluación anatómica detallada 3D/4D de columna

o Evaluación 3D/4D de adherencia placentaria anormal

- Evaluación 3D/4D de anatomía o malformaciones uterinas

o Evaluación 3D/4D de la actitud y conducta fetal fomentando el apego emocional.

- Modo ecográfico:

- 2D para biometría, crecimiento y anatomía. Un buen conocimiento y evaluación en $2 D$ es requisito para realizar un $3 D-5 D$ de calidad.

o Doppler (Angio 3D-HB flow, y otros sub modos) para evaluación de vasos fetales o placentarios.

o 3D-5D optimiza la evaluación anatómica y de anomalías en múltiples planos o evaluación vascular tridimensional.

- Abordaje: Transabdominal y complementariamente transvaginal, para la evaluación de estructuras fetales de acceso vaginal o durante el primer trimestre.

- Duración: Aproximadamente 50 minutos si es un solo feto.

Financiamiento: Autofinanciado.

Conflicto de interés: Los autores declaran no tener algún conflicto de intereses. 


\section{REFERENCIAS BIBLIOGRÁFICAS}

1. Committee on Practice Bulletins Obstetrics and the American Institute of Ultrasound in Medicine. Practice bulletin No. 175: Ultrasound in pregnancy. ObstetGynecol.2016;128(6):e241 e256.doi:10.1097/AOG.0000000000001815.

2. Wax J, Minkoff $\mathrm{H}$, Johnson A, et al. Consensus report on the detailed fetal anatomic ultrasound examination: indications, components, and qualifications. J Ultrasound Med 2014; 33:189-195.

3. American College of Radiology. ACR-ACOG-AIUMSRU practice parameter for the performance of obstetrical ultrasound. Reston (VA): ACR; 2013. Available at: http:// www.acr.org/ /media/ACR/Documents/PGTS/ guidelines/ US_Obstetrical.pdf. Accedido Febrero 24, 2019.

4. American Institute of Ultrasound in Medicine. AIUM practice guideline for the performance of obstetric ultrasound examinations. J Ultrasound Med 2013;32: 1083-101.

5. Salomon LJ, Alfirevic Z, Bilardo CM, Chalouhi GE, Ghi T, Kagan KO, Lau TK, Papageorghiou AT, Raine-Fenning NJ, Stirnemann J, Suresh S, Tabor A, Timor-Tritsch IE, Toi A, Yeo G. ISUOG practice guidelines: performance of first-trimester fetal ultrasound scan. Ultrasound Obstet Gynecol 2013; 41: 102-113.

6. Salomon LJ, Alfirevic Z, Berghella V, Bilardo C, HernandezAndrade E, Johnsen SL, et al. Practice guidelines for performance of the routine mid-trimester fetal ultrasound scan. ISUOG Clinical Standards Committee. Ultrasound Obstet Gynecol 2011; 37:116-26

7. Bhide A, Acharya G, Bilardo CM, et al. ISUOG practice guidelines: use of Doppler ultrasonography in obstetrics. Ultrasound Obstet Gynecol 2013; 41:233-239. doi: 10.1002/ uog. 12371.

8. Mari G, Norton ME, Stone J, Berghella V, Sciscione AC, Tate D, et al. Society for Maternal-Fetal Medicine (SMFM) Clinical Guideline \# 8: the fetus at risk for anemia-diagnosis and management. Society for Maternal-Fetal Medicine (SMFM). Am J Obstet Gynecol 2015;212:697-710.

9. Method for estimating due date. Committee Opinion No. 611. American College of Obstetricians and Gynecologists. Obstet Gynecol 2014;124:863-6.
10. Abuhamad AZ, Benacerraf BR, Woletz P, Burke BL. The accreditation of ultrasound practices: impact on compliance with minimum performance guidelines. J Ultrasound Med 2004;23:1023-9.

11. Wax J, Minkoff $H$, Johnson A, et al. Consensus report on the detailed fetal anatomic ultrasound examination: indications, components, and qualifications. J Ultrasound Med 2014;33(02): 189-195.

12. ISUOG Education Committee recommendations for basic training in obstetric and gynecological ultrasound. Ultrasound Obstet Gynecol 2013:1-4.

13. American Institute of Ultrasound in Medicine Obstetric (Trimester-Specific \& Adjunct Detailed Fetal Anatomic US)

14. Society of Diagnostic Medical Sonography

15. American Congress of Obstetricians \& Gynecologists

16. The fetal medicine foundation https://fetalmedicine.org/fmfcertification/certificates-of-competence

17. Obstetric Ultrasound Guidelines Version 7 March 2011. Harris Birthright Research Centre for Fetal Medicine King's College Hospital

18. Council ACR. ACR-ACOG-AIUM-SRU Practice Parameter for the Performance of Obstetrical Ultrasound. https://www.acr. org/-/media/ACR/Files /Practice-Parameters/us-ob.pdf?la=en Revised and published October 1, 2013. Acceso Enero 27, 2019.

19. Professional practice guidelines and policy statements for canadian sonography.http://www.sonographycanada. ca/ A p ps/Sites - Management/FileDownload/ DataDownload/46650/SC_ProfPractice\%20Eng\%20Rev\%20 03Feb2017\%20final/pdf/1/1033

20. The American Registry for Diagnostic Medical Sonography (ARDMS) http://www.ardms.org/Discover-ARDMS/careersin-sonography/Pages/default.aspx

Correspondencia: Aldo Benel Chamaya.

E-mail: walcasurq@yahoo.es 


\section{ANEXOS}

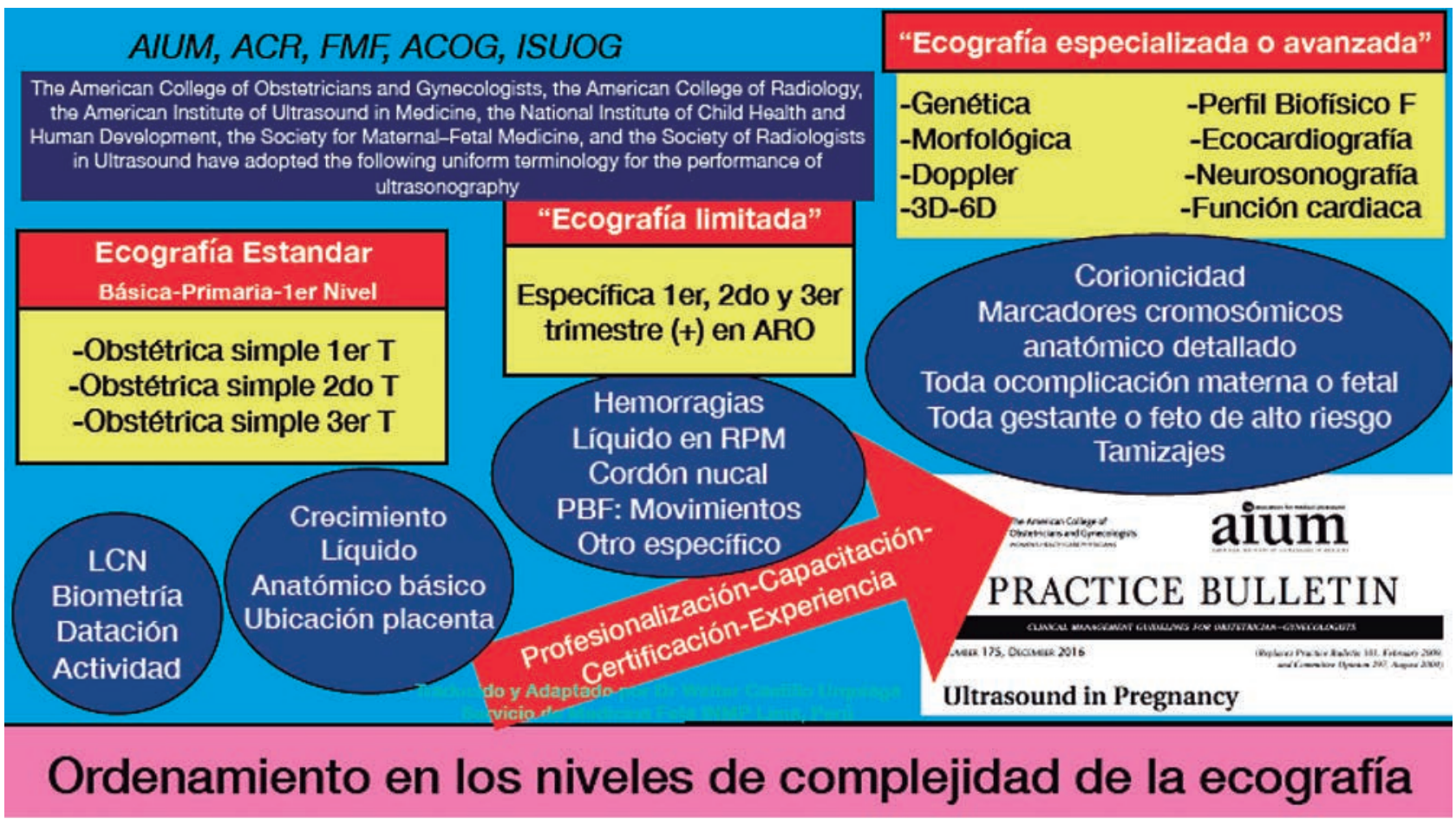

Figura 1: Tipos de ecografía según nivel de complejidad

1. Ultrasound in pregnancy. ObstetGynecol.2016;128(6):e 241 e256 
Tabla 1. Componentes de la evaluación ecoráfica básica y detallada.

\section{COMPONENTES DE LA EVALUACIÓN ECOGRÁFICA BÁSICA Y DETALLADA}

J Ultrasound Medicine 2014

Consensus Report of the Detailed fetal anatomic ultrasound examination AIUM, SMFM, ACOG, ACR, SRU

\begin{tabular}{|c|c|c|}
\hline COMPONENTES & BÁSICO & DETALLADO \\
\hline \multirow{8}{*}{ PRIMER TRIMESTRE } & $\begin{array}{l}\text { Longitud corono nalga } \\
\text { Actividad cardiaca } \\
\text { Placenta }\end{array}$ & $\begin{array}{l}\text { Translucencia nucal y marcadores } \\
\text { Anatomía y biometría } \\
\text { Doppler Uterinas }\end{array}$ \\
\hline & Ventrículos laterales & 3er ventrículo ${ }^{a}$ \\
\hline & Plexo coroideo & 4to ventrículo \\
\hline & Linea media & $V^{\text {Ventrículos laterales }}{ }^{b}$ \\
\hline & Cavum septum pelucidum & Lóbulos cerebelosos, Vermis y Cisterna Maga ${ }^{b}$ \\
\hline & Cerebelo & Cuerpo calloso ${ }^{a}$ \\
\hline & \multirow[t]{2}{*}{ Cisterna Magna } & Integridad y forma de la bóveda craneal \\
\hline & & Parénquima cerebral Cuello \\
\hline \multirow{5}{*}{ CARA } & \multirow{5}{*}{ Labio superior } & Perfil \\
\hline & & Cara en coronal (labios/nariz/cristalinos ${ }^{a}$ ) \\
\hline & & Paladara, maxilar, mandíbula y lengua ${ }^{a}$ \\
\hline & & Tamaño y posición de orejas ${ }^{a}$ \\
\hline & & Órbitas ${ }^{a}$ \\
\hline \multirow{6}{*}{ TÓRAX Y CORAZÓN } & \multirow{6}{*}{$\begin{array}{l}\text { Actividad cardiaca } \\
\text { Vista de } 4 \text { cámaras } \\
\text { Salida del Ventrículo izquierdo } \\
\text { Salida del ventrículo derecho }\end{array}$} & Arco aórtico \\
\hline & & Vena cava superior e inferior \\
\hline & & Vista de 3 vasos \\
\hline & & Vista de 3 vasos tráquea \\
\hline & & Pulmones \\
\hline & & Integridad del diafragma \\
\hline \multirow{7}{*}{ ABDOMEN } & Estómago (presencia, tamaño y sitio) & Intestino delgado y grueso ${ }^{a}$ \\
\hline & Riñones & Glándulas adrenales ${ }^{a}$ \\
\hline & Vejiga urinaria & Vesícula $^{a}$ \\
\hline & Inserción del cordón en el abdomen & Hígado \\
\hline & Número de vasos del cordón & Arterias renales ${ }^{a}$ \\
\hline & & $\mathrm{Bazo}^{\mathrm{a}}$ \\
\hline & & Integridad de pared abdominal ${ }^{\mathrm{b}}$ \\
\hline
\end{tabular}




\section{COMPONENTES DE LA EVALUACIÓN ECOGRÁFICA BÁSICA Y DETALLADA}

J Ultrasound Medicine 2014

Consensus Report of the Detailed fetal anatomic ultrasound examination AIUM, SMFM, ACOG, ACR, SRU

\begin{tabular}{|c|c|c|}
\hline COMPONENTES & BÁSICO & DETALLADO \\
\hline COLUMNA VERTEBRAL & $\begin{array}{l}\text { Cervical } \\
\text { Lumbar }\end{array}$ & $\begin{array}{l}\text { Integridad de columna y tejido blando } \\
\text { suprayacente }^{\text {b }} \\
\text { Forma y curvatura de columna }\end{array}$ \\
\hline \multirow[b]{2}{*}{ EXTREMIDADES } & Piernas & Número, arquitectura y posición \\
\hline & Brazos & Manos Pies \\
\hline GENITALES & En gestaciones múltiples & $\begin{array}{l}\text { Dedos: Número y posición }{ }^{a} \\
\text { Sexo }^{\mathrm{a}}\end{array}$ \\
\hline \multirow[t]{2}{*}{ PLACENTA } & Cuando esté medicamente indicado & \\
\hline & Localización y grado & Masas \\
\hline \multirow{7}{*}{ EVALUACIÓN RUTINARIA } & Relación al Orificio cervical interno & Inserción del cordón en Placenta \\
\hline & Apariencia & \multirow{2}{*}{$\begin{array}{l}\text { Lóbulo accesorio/subcenturiado con localización } \\
\text { del suministro vascular a la placenta principal }{ }^{a}\end{array}$} \\
\hline & Inserción del cordón en la Placenta (si es posible) & \\
\hline & Número fetal & \\
\hline & Presentación & \\
\hline & Estimación semicuantitativa del líquido, ILA & \\
\hline & Cérvix (transvaginal cuando esté indicado) & \\
\hline \multirow{3}{*}{ ANATOMIA MATERNA } & Útero Anexos & \\
\hline & Diámetro biparietal & Cerebelo $^{a}$ \\
\hline & Circunferencia cefálica & Diámetro orbitario interno y externo ${ }^{a}$ \\
\hline \multirow[t]{3}{*}{ BIOMETRIA } & Circunferencia abdominal & Pliegue nucal \\
\hline & Longitud del Fémur & Medición del Hueso nasal \\
\hline & Peso estimado Fetal y Percentil peso & Húmero ${ }^{\mathrm{a}}$ Cúbito/Radio ${ }^{\mathrm{a}}$ Tibia/Peronéa \\
\hline
\end{tabular}

